\title{
Comercio electrónico y marketing digital en tiempos de Covid-19. Análisis en una empresa privada peruana
}

\section{E-commerce and digital marketing in times of Covid-19. Analysis in a peruvian private company}

\begin{abstract}
RESUMEN
Uno de los grandes desafíos de las empresas del sector privado en la pandemia del Covid-19, es comprender el comportamiento del comercio electrónico, ya que de esta forma podrán obtener mejoras en su economía, haciendo uso de medios publicitarios para poder ampliar el nivel de ventas $\mathrm{y}$ visitas de los clientes y mejorar su marketing digital. El presente estudio busca determinar si el comercio electrónico influye en el marketing digital en una empresa privada de Perú. El enfoque fue cuantitativo, de tipo aplicada. La población se vio representada por 1200 clientes y la muestra por un total de 291. La técnica de recojo de datos fue la encuesta y el instrumento los Cuestionarios. Los resultados demostraron que, el comercio electrónico y el marketing digital tienen un nivel regular, debido a que con poca frecuencia se toman acciones referentes a este medio. Se concluyó que el comercio electrónico influye en la mejora del marketing digital, comprendiendo que cuando la empresa privada analizada, tenga en cuenta en sus políticas hacer uso de estas herramientas obtendrá indicadores satisfactorios en la captación de clientes y por ende en el nivel de ventas.
\end{abstract}

Palabras Clave: Comercio electrónico; marketing digital; interacción; difusión de contenidos; retención de clientes.

\begin{abstract}
One of the great challenges for private sector companies in the Covid-19 pandemic is to understand the behavior of electronic commerce, since in this way they will be able to obtain improvements in their economy, making use of advertising media to be able to expand the level of sales and customer visits, rather, improve your digital marketing. This study seeks to determine if electronic commerce influences digital marketing in a private company in Peru. The approach was quantitative, applied type. The population was represented by 1200 clients and the sample by a total of 291. The data collection technique was the survey and the instrument the questionnaires. The results showed that electronic commerce and digital marketing have a regular level, due to the fact that actions related to this
\end{abstract}

\section{Sonia Francisca Soler Grillo}

soniasolerg@hotmail.com

Universidad Peruana de Ciencias Aplicadas. Lima, Perú

Presentado: 29/05/2021 - Aceptado: 31/08/2021 - Publicado: 23/12/2021

(C) Los autores. Este artículo es publicado por la revista Gestión en el Tercer Milenio de la Facultad de Ciencias Administrativas Universidad Nacional Mayor de San Marcos. Este es un artículo de acceso abierto, distribuido bajo los términos de la licencia Creative Commons Atribución 4.0 Internacional (CC BY 4.0) [https://creativecommons.org/licenses/by/4.0/deed.es] que permite el uso, distribución y reproducción en cualquier medio, siempre que la obra original sea debidamente citada de su fuente original. 
medium are rarely taken. It was concluded that electronic commerce influences the improvement of digital marketing, understanding that when the analyzed private company takes into account in its policies to make use of these tools, it will obtain satisfactory indicators in the acquisition of clients and therefore in the level of sales.

Keywords: Electronic commerce; digital marketing; interaction; content dissemination; client retention.

\section{INTRODUCCIÓN}

En la actual era digital, el comercio electrónico y el marketing digital se han convertido en herramientas fundamentales para el crecimiento de las empresas. El marketing digital es una nueva técnica en el campo del marketing para generar un impacto en los consumidores a través de una amplia gama de canales de interacción (redes sociales, aplicaciones móviles, blogs, correos electrónicos, optimizadores de motores de búsqueda), orientados a generar una comunicación más cercana con los consumidores. Además, el marketing digital implica la promoción de bienes y servicios a través de una plataforma de comercio electrónico con respuesta en tiempo real mediante una conexión a Internet. En este sentido, el marketing digital forma un subconjunto del comercio electrónico, lo cual implica que, el comercio electrónico podría mejorar el marketing, expandir el mercado tanto a nivel nacional como internacional, aumentar la demanda, mantener y desarrollar relaciones con distribuidores, clientes y proveedores de forma rápida y puntual (Bala \& Verma, 2018).

No obstante, aunque el comercio electrónico es importante, este se desarrolla aún de manera lenta en el mundo, como en Lituania que es poca la participación de las compras en línea sobre todo en los minoristas, llegando solo al $3.5 \%$ en 2016 (Išoraitė \& Miniotienè, 2018). 0 en Turquía que también este tipo de comercio se viene desarrollando de manera lenta (Basarir \& Mardikyan, 2017). Esto puede que afecte a la calidad del marketing, ya que algunas empresas aún mantienen el marketing tradicional, generalmente por costumbre de las generaciones anteriores (Farida, et al., 2017). No obstante, la pandemia del Covid-19, ha generado cambios en la actividad económica, no solo por las medidas sanitarias adoptadas, sino con la adopción del comercio electrónico ya que como señala Bhatti et al. (2020) a nivel mundial el $52 \%$ de personas evita salir a comprar en zonas concurridas, lo cual genera mayores ventas en la modalidad de e-commerce hasta en un 74\% en todo el mundo, en distintas plataformas virtuales.

A nivel nacional, se evidencia que algunas empresas todavía no emplean el comercio electrónico. Como los productores pyme de calzados de la ciudad de Trujillo, que no están capacitados en esta clase de comercio, llegando a un nivel bajo de conocimientos en un $85 \%$. Debido a ello, no saben cómo emplear esta herramienta para incrementar sus ventas, originándoles problemas como bajos ingresos y la absorción por la competencia (Mucha, 2018,). En todo el Perú, el análisis de Blacksip (2019) demuestra que el e-commerce en el país va introduciéndose en un $72,9 \%$, pero la pandemia ha acelerado este proceso, ya que hubo un movimiento de dinero de hasta 10 millones de nuevos soles a inicios de la pandemia, posteriormente llegando a los 700 millones en el transcurso de la misma, habiendo una demanda considerable para los negocios en general y para los que entran al e-commerce (Fuentes, 2020). Aquello puede favorecer al marketing de las mismas, pero esta tiene que ser manejada de manera adecuada, de lo contrario no resulta favorable.

En el ámbito local, el problema del comercio electrónico se pudo comprender a través de entrevistas informales no estructuradas a los clientes de una empresa peruana que hace uso de este tipo de comercio, la cual difunde su contenido por medio de las redes sociales, correos electrónicos y la web. No obstante, las campañas publicitarias no han logrado obtener los resultados esperados, ya que la página web de la empresa no tuvo las visitas esperadas y el nivel de ventas no se ha incrementado como se había estimado. Asimismo, se ha observado que esta situación se debería a que la estructura de la página web, resulta difícil de entender para los 
usuarios, el contenido no es comprensible para ellos y consideran que se ve desarmonizado. Por otro lado, han manifestado que mientras intentan realizar una compra tienen bugs (errores del sitio web) por ejemplo, vuelven a la página de inicio cuando dan clic para ver un producto, les sale error en su compra, se carga el doble de la cantidad de productos en su canasta y, en algunos casos han tenido un cobro duplicado por cosas que no adquirieron.

Todos esos problemas estarían afectando al marketing digital de la empresa, reduciendo sus ventas y ganando una mala reputación en este tipo de comercio, por tal motivo es necesario profundizar en esta situación, sobre todo en estos momentos de crisis por la Covid-19, que genera en las empresas una necesidad de ser más competentes estando a la par con la tecnología. Por tanto, el presente estudio tuvo como objetivo determinar la influencia del comercio electrónico para mejorar el marketing digital en una empresa privada en el Perú.

Después de examinar la problemática del comercio electrónico y el marketing digital, se llegó a observar que existe un grupo significativo de estudios que guardan una estrecha relación con el propósito de investigación a nivel nacional, iniciando por Matas et al., (2016) con su estudio que tuvo como objetivo analizar el comercio electrónico en una empresa en Junín, considerando como población a 62 entidades, en donde concluye que los encuestados no conocen los mercados electrónicos como medio para la internacionalización, el $82.35 \%$ de las empresas no operan en los mercados electrónicos y del porcentaje que sí operan en los mercados electrónicos, solo el $66.67 \%$, consideran que es un herramienta de promoción de sus productos y de contacto con el cliente, considerando que existe espacio para aprovechar las ventajas del comercio electrónico para aumentar la eficacia del marketing a través de los medios digitales. De igual manera, Mucha (2018) en su estudio sobre el comercio electrónico y su relación con el nivel de ventas de 78 Pymes productoras de calzado, de la Provincia de Trujillo, encontró que el $85 \%$ de los encuestados tiene un bajo nivel de conocimiento del comercio electrónico y el $71 \%$ se muestra predispuesto a capacitarse en este tipo de comercio, concluyendo que las empresas no emplean el comercio electrónico debido a la inseguridad en su uso y al desconocimiento de sus procesos.

A nivel internacional, Farida et al. (2017) en su análisis en 41 empresas, encuentra que existe influencia del comercio electrónico en el marketing de la empresa. Por otro lado, Hudák et al. (2017) en amplio análisis de seis países, pudo encontrar que cerca del $70 \%$ de empresas hacen uso del e-mail marketing para su publicidad y consideran que el comercio electrónico es importante para hacer su publicidad, porque permite llegar a más clientes. Finalmente, Rungsrisawat et al. (2019) encuentra encuestando a 319 compradores que el 59\% piensa en comprar por Internet en redes sociales como Facebook, Twitter, Linkedin, Instagram, etc., para ellos son las principales fuentes para conseguir información.

\section{Comercio electrónico}

De acuerdo con el sustento teórico de Kütz (2016) el comercio electrónico es el intercambio de bienes y servicios entre organizaciones independientes y/o personas apoyado en el uso integral de un potente sistema de TIC y de una infraestructura de red estandarizada a nivel mundial, para lo cual, los socios comerciales tienen que adaptar sus procesos de negocios y su sistema TIC. Por su parte Villa et al. (2018) analizaron el comercio electrónico desde cuatro perspectivas. Desde el punto de vista de la comunicación, el comercio electrónico es la entrega de información, bienes o pagos efectuados mediante líneas telefónicas, redes informáticas o medios similares. Desde la óptica del proceso empresarial, los autores lo definen como la aplicación de tecnología para alcanzar la automatización de las transacciones o flujo de trabajo. Desde la perspectiva del servicio, se define como un instrumento para alcanzar los objetivos de la empresa y del cliente, de tal modo que se administren de manera adecuada los costos mejorando de una manera significativa los tiempos de entrega. Por último, desde el enfoque en línea, el comercio electrónico permite ver y observar productos e información de internet y demás servicios por la web.

El comercio electrónico se evalúa en base al modelo propuesto por Oliveira et al. (2017) que consideran cuatro fuentes de la confianza 
del consumidor hacia el comercio electrónico. Dichas fuentes inciden en las dimensiones de la confianza del consumidor (competencia, integridad y benevolencia), es decir, sobre las percepciones de un proveedor de Internet. A su vez, las dimensiones inciden en la confianza general del consumidor, repercutiendo en la intención de compras en línea. Las cuatro fuentes son: características del consumidor; características de la empresa; infraestructura del sitio web e interacción.

\section{Marketing digital}

En cuanto al marketing digital, se considera a Kannan y Li (2017) quienes hacen énfasis, de que se trata de un proceso adaptativo habilitado por la tecnología a través del cual las empresas cooperan con los clientes y socios para generar, comunicar, brindar y mantener valor para todas las partes interesadas. Esto además quiere decir, que el marketing digital genera valor de varias maneras mediante nuevas experiencias del cliente y nuevas interacciones. Asimismo, el marketing digital se habilita por un conjunto de puntos de contactos digitales adaptables, que abarcan la actividad del marketing, las instituciones, los procesos y los clientes.

En tanto que, Saura (2020) definió el marketing digital como una serie de técnicas generadas en internet para persuadir a los usuarios a comprar un producto o servicio. Asimismo, de acuerdo al autor, entre las técnicas de marketing digital más empleadas actualmente se encuentran: la optimización de motores de búsqueda (SEO), es decir, la optimización de los resultados de búsqueda de los principales motores de búsqueda; Search Engine Marketing (SEM) o publicidad programática, esto es, estrategias para patrocinar anuncios en motores de búsqueda o en espacios publicitarios en banners en sitios web; así como Social Media Marketing (SMM), es decir, estrategias de interacción con los usuarios en las redes sociales a través de anuncios.

Para realizar la evaluación respectiva de la variable marketing digital se han identificado tres dimensiones: visibilidad, difusión de contenido y retención de clientes. La visibilidad, es la capacidad de una marca, institución, empresa o profesional para ser visualizado y reconocido por la mayor cantidad de usuarios, en especial, por aquellos que son parte del grupo objetivo. Asimismo, en cuanto al posicionamiento en buscadores, puede ser asociada con la facilidad para encontrarse. La visibilidad depende de una variedad de factores como la reputación, la imagen, etc., que pueden incidir en el grado de visibilidad del usuario, esta cuenta con dos indicadores: redes sociales y página web (Piñeiro \& Martínez, 2016).

\section{MÉTODOS}

La presente investigación se encuentra dentro del enfoque cuantitativo, el cual es denominado como positivista o tradicional, que determina un atributo esencial en examinar los datos, enfocándose en la problemática en estudio, la cual tiene como propósito generalizar los hallazgos, por tanto, el estudio busca realizar una evaluación general de las variables y generar la presentación de los resultados a fin de tomar decisiones correctas (Bernal, 2016). Por otra parte, el tipo de estudio fue aplicado, ya que brinda una solución inmediata al problema a tratar, recomendando así las soluciones en relación al comercio electrónico para mejorar el marketing digital de una empresa peruana (Baimyrzaeva, 2018). Dentro de su diseño, el estudio fue no experimental, debido a que no hubo manipulación deliberada de las variables (Reiro, 2016) y se consideró transversal porque solamente se recoge datos en un solo momento (Kumar, 2011).

El estudio se realizó en Lima, en una empresa privada, siendo la unidad de información el número de usuarios que suelen adquirir los productos de la empresa, a través de un proveedor en línea y que realizan el pago por medio de tarjetas de créditos o Paypal. Estos en conjunto suman un total de 1200 clientes, pero mediante la formula del muestreo aleatorio simple al 95\% de confianza, se determinó una muestra de 291 clientes que poseen edades entre los 18 a 30 años. Para recopilar la información, se empleó como técnica la encuesta y como instrumento el cuestionario, herramientas para ambas variables que son estructuradas en base a un formulario de preguntas (ítems) para dar respuesta a los objetivos de estudio, (Bernal, 2016). Dichos instrumentos fueron validados mediante el juicio 
de expertos, participando 3 especialistas en el tema, los cuales valoraron cada ítem según la siguiente escala: $1=$ muy deficiente, $2=$ deficiente, $3=$ =aceptable, $4=$ buena, $5=$ excelente. Además, para la confiabilidad se aplicó de la prueba estadística Alfa de Cronbach, gracias a los datos de una muestra piloto de 30 clientes de una empresa privada, encontrando para la variable comercio electrónico un coeficiente de 0.962 y para el marketing digital 0.812 , por tanto, los instrumentos son confiables debido a que cuanto más cerca al 1 presentan mayor confiabilidad (Corral, 2009).

El análisis e interpretación de la información obtenida a través de la aplicación de los cuestionarios, se realizó en tres fases. En primer lugar, se generó una base de datos empleando el programa Microsoft Excel, información que se trasladó al software estadístico SPSS vs24, en donde se codificaron los datos. En segundo lugar, se realizó un análisis descriptivo de los datos a través del cálculo de la media, promedio, mediana, varianza, valor máximo, mínimo, etc. Y finalmente se estableció la influencia por medio de una prueba de regresión lineal, empleando el $\mathrm{R}^{2}$, luego de corroborar que los datos tengan distribución normal.

\section{RESULTADOS}

\section{Resultados descriptivos}

En el presente apartado se describen los resultados de los estadísticos descriptivos de las variables y dimensiones del estudio, así como sus frecuencias y porcentajes, que fueron determinados mediante una fórmula sencilla para clasificar sus niveles. De este modo, se obtuvo para la variable comercio electrónico una media de 94,6048, así como una mediana de 93 y una moda de 100. La desviación estándar fue de 32,07786, la varianza de 1028,989, y un valor mínimo de 35 y máximo de 161. Por otro lado, según la Tabla 1, se aprecian los niveles en que se encuentra el comercio electrónico, siendo en su mayoría regular al $47 \%$, lo cual indica que la mayoría de los clientes, considera que el comercio electrónico es regularmente adecuado en la empresa teniendo ciertas deficiencias.
Tabla 1

Comercio electrónico

\begin{tabular}{ccccc}
\hline \multirow{2}{*}{ Calificación } & \multicolumn{2}{c}{ Rango } & Frec. & \% \\
& Desde & Hasta & & \\
\hline Deficiente & 33 & 77 & 91 & $31 \%$ \\
Regular & 78 & 122 & 136 & $47 \%$ \\
Eficiente & 123 & 165 & 64 & $22 \%$ \\
Total & & & $\mathbf{2 9 1}$ & $\mathbf{1 0 0 \%}$ \\
\hline
\end{tabular}

Nota. Elaboración propia.

Con respecto al marketing digital, los datos descriptivos obtenidos, demuestran que existe una media de 46,3230, así como una mediana de 45 y una moda de 43. La desviación estándar fue de 15,31797, la varianza de 234,640, con un valor mínimo de 17 y máximo de 79. Además, tal como muestra la Tabla 2, el marketing digital, en su mayoría es regular al $48 \%$, indicando que la mayoría de los clientes perciben de manera regular que la empresa emplea estrategias para ser visible, difundir el contenido y retener a los clientes por medio del marketing digital.

Tabla 2

Marketing digital

\begin{tabular}{ccccc}
\hline \multirow{2}{*}{ Calificación } & \multicolumn{2}{c}{ Rango } & Frec. & \% \\
& Desde & Hasta & & \\
\hline Deficiente & 16 & 37 & 86 & $30 \%$ \\
Regular & 38 & 60 & 141 & $48 \%$ \\
Eficiente & 61 & 80 & 64 & $22 \%$ \\
Total & & & $\mathbf{2 9 1}$ & $\mathbf{1 0 0 \%}$ \\
\hline
\end{tabular}

Nota. Elaboración propia.

\section{Resultados inferenciales}

Para el análisis inferencial, primero se determinó la normalidad de los datos, empleando la prueba de Kolmogorov-Smirnov, debido a que la muestra es superior a 50 individuos, tal como se muestra en la Tabla 3, la significancia es superior a 0.05 , indicando que los datos están distribuidos según la curva normal, de lo cual se determina que la prueba a emplear para conocer la influencia de la variable comercio electrónico sobre el marketing digital, fue una prueba paramétrica, siendo la prueba de regresión lineal, considerando el $\mathrm{R}^{2}$ para conocer la fuerza de dicha influencia. 
Tabla 3

Prueba de normalidad Kolmogorov-Smirnov ${ }^{a}$

\begin{tabular}{cccc}
\hline & Estadístico & gl & Sig. \\
\hline Comercio electrónico & 0,045 & 384 & $0,200^{*}$ \\
Marketing digital & 0,052 & 291 & 0,058 \\
\hline
\end{tabular}

Nota. a. Corrección de significancia de Lilliefors. Elaboración propia.

De acuerdo con los resultados del objetivo principal, el coeficiente del $\mathrm{R}^{2}$ demuestra que el comercio electrónico influencia 99,0\% en el marketing digital como se describe a detalle en la Tabla 4.

\section{DISCUSIÓN}

El comercio electrónico, posee en su mayoría una percepción regular por parte de los clientes, lo cual indica que regularmente están orientados a comprar en la marca, además que de manera regular la imagen, la reputación y confianza prevalece en la empresa privada. Así también, posee un sitio web relativamente bien estructurado en el que se puede interactuar. Este resultado se asemeja al estudio de Rungsrisawat et al. (2019), quien refiere que la gran mayoría de encuestados considera que algunas plataformas como Facebook o Twitter, entre otras, son ideales para comprar, por tanto, las empresas que están activas en estas plataformas aplican el comercio electrónico, y en el presente estudio la empresa privada lo hace regularmente. Además, como refiere Matas et al. (2016), en su estudio, el $82.35 \%$ de las empresas no operaba en los mercados electrónicos, y que más del $66 \%$ no promociona sus productos y servicios por este medio, por tanto, los clientes no se contactan casi con ellos.

La variable marketing digital que responde al segundo objetivo, posee en su mayoría una percepción regular por parte de los clientes, comprendiendo que regularmente la empresa prevalece en el marketing digital que aplica, siendo regularmente visible y es regular la difusión de su contenido, además de que los clientes a veces recomiendan y difunden su lo que ofrece. Este resultado, se compara con el de Hudák et al. (2017), que refiere que cerca de $70 \%$ de las empresas emplean el e-mail marketing para realizar publicidad, por tanto, aún no emplean en su totalidad o gran parte de esta estrategia, lo cual estaría generando que su marketing no sea el adecuado desde la perspectiva de los clientes.

Con respecto al objetivo general, se encontró que el comercio electrónico influye en el marketing digital en un 99,0\% según el coeficiente de determinación (R cuadrado), entendiendo que cuando la empresa emplea diversas acciones y estrategias relacionadas a la venta de sus productos vía online, su marketing será mucho mejor. Este resultado se asemeja al de Farida et al. (2017) quien establece una influencia entre las variables de estudio ( $\mathrm{t}=5.217)$, concluyendo que la práctica del comercio electrónico permitiría ampliar la gama de marketing de las pymes, aumentar la demanda, mantener y desarrollar relaciones con distribuidores, clientes y proveedores de forma rápida y puntual. Así también el de Hudák et al. (2017) que llegó a concluir que el comercio electrónico es importante para la implementación de actividades de marketing, ya que permite llegar a un número de usuarios mucho mayor que el marketing clásico.

De acuerdo a lo mencionado, se entiende que el modelo de Chen y Dhillon que refiere Oliveira et al. (2017), ayuda a comprender cómo los componentes de entrada, los cuales son las dimensiones analizadas, son aspectos fundamentales, que al ser trabajados por parte de la empresa, generan la confianza que el consumidor necesita, para seguir adquiriendo los productos y servicios de la empresa, lo cual a su vez refuerza el marketing digital de la misma. De esto se entiende que los altos niveles de marketing, conllevan un vínculo de confianza con el consumidor.

Tabla 4

Resumen del Modelo (Comercio electrónico)

\begin{tabular}{ccccc}
\hline Modelo & $\mathbf{R}$ & R cuadrado & R cuadrado ajustado & Error estándar de la estimación \\
\hline 1 & $0,995^{\mathrm{a}}$ & 0,990 & 0,990 & 1,50307 \\
\hline
\end{tabular}

Nota. a. Predictores: (Constante), Comercio electrónico. Elaboración Propia 


\section{CONCLUSIONES}

El comercio electrónico influye en mejorar el marketing digital en una empresa privada en Perú, de igual manera sucede con sus componentes o dimensiones, es por ello que cuando la empresa desarrolle mejor este tipo de comercio y lo use recurrentemente, afectará de manera positiva el marketing digital de la misma, llegando a ser más visible y atrayendo a nuevos clientes, sobre todo en estos tiempos de pandemia del Covid-19, en que las personas realizan mayores compras en línea por evitar el contacto físico debido al contagio.

Por otro lado, se encontró que los niveles de comercio electrónico y marketing digital en la empresa de estudio, se encuentran regulares, indicando que los clientes tienen una percepción regular de la empresa, de igual manera en su reputación y confianza hacia ella, además de que el entorno web de la empresa está regularmente estructurado para interactuar y consumir. También, la empresa es regularmente visible, además de que a veces difunde su contenido y los clientes lo recomiendan de manera regular.

Y finalmente, se pudo corroborar que el modelo de Chen y Dhillon, demuestra que la mejora de los componentes del comercio electrónico, repercute no solo en la confianza del consumidor, sino en el marketing digital, siendo un factor influyente en esta variable, del cual se infiere además, que un buen marketing se basa en la confianza que tiene el consumidor con la marca.

Como recomendaciones, se invita a los encargados del área comercial y de marketing de la empresa estudiada, que trabajen de manera más articulada, controlando las estrategias de comercio electrónico que aplican para realizar los cambios requeridos y mejorarlos. Así también, desarrollar encuestas breves dentro de las plataformas virtuales que emplean, para recopilar la opinión del servicio brindado y las posibles mejoras por parte de los clientes. A los encargados del área de marketing, se les recomienda realizar una inversión adicional para publicitar en las plataformas más conocidas como Facebook, Instagram, etc., además automatizar la mensajería en estos para resolver dudas básicas en relación a la compra de algún producto o servicio. Por otro lado, para el área de comercio, evaluar continuamente la información obtenida de las estadísticas propias de las plataformas ya conocidas que aportan a este fin y mejorar el sistema de información de la propia página web de la empresa, para conocer patrones de compra y su rendimiento, de tal forma que se tomen decisiones más certeras en base a dicha información. Finalmente, implementar mejor la página web de la empresa para que sea más accesible, entendible, amigable y segura en el proceso de compra, además de más práctica y sencilla.

\section{REFERENCIAS BIBLIOGRÁFICAS}

Bala, M., \& Verma, D. (2018). A Critical Review of Digital Marketing [Una revisión crítica del marketing digital]. International Journal of Management, IT \& Engineering, 8(10), 321339. https://www.researchgate.net/publication/328253026_A_Critical_Review_of_Digital_Marketing.

Bhatti, Anam \& Akram, Hamza \& Basit, Hafiz \& Khan, Ahmed \& Mahwish, Syeda \& Naqvi, Raza \& Bilal, Muhammad. (2020). E-commerce trends during COVID-19 Pandemic. International Journal of Future Generation Communication and Networking. 13.

Baimyrzaeva, M. (2018). Begginers'guide for applied research process: what is it, and why and how to do it? (Ocasional paper, $\mathrm{N}^{\circ} 4$ ). https://www. ucentralasia.org/Content/Downloads/UCAIPPA-OP4-Beginners $\% 20$ Guide $\% 20$ for $\% 20$ Applied\%20Research\%20Process-Eng.pdf

Basarir, B., \& Mardikyan, S. (2017). Factors affecting E-commerce adoption: A case of Turkey [Factores que afectan la adopción del comercio electrónico: un caso de Turquía]. The International Journal of Management Science and Information Technology (IJMSIT)(23), 1-11. https://www. econstor.eu/bitstream/10419/178834/1/IJMSIT_y2017_i23_p001-011.pdf

BlackSip. (2019). Reporte de Industria: El eCommerce en Perú 2019. https://content.blacksip. com/ebook-reporte-de-industria-el-ecommerce-en-peru-2019

Bernal, C. (2016). Metodología de la investigación: Administración, Economía, Humanidades y Ciencias Sociales.(4ta ed.) Pearson Editorial.

Corral, Y. (2009). Validez y confiabilidad de los instrumentos de investigacion para la recoleccion de datos. Revista ciencia de la educación, 19(33), 228-247. https://es.scribd.com/ 
doc/316036286/Validez-y-Confiabilidad-Para-Los-Instrumentos-de-Recoleccion-de-Datos

Farida, N., Naryoso, A., \& Yuniawan, A. (2017). Model of Relationship Marketing and E-Commerce in Improving Marketing Performance of Batik SMEs [Modelo de marketing relacional y comercio electrónico para mejorar el rendimiento de marketing de las pymes batik]. Jurnal Dinamika Manajemen, 8(1), 20-29. https://journal.unnes.ac.id/nju/index.php/jdm/article/ view/10408/6438

Fuentes, H. (2020). Crecimiento del e-commerce en el Perú en época de pandemia. https://www.datatrust.pe/ecommerce/ecommerce-en-el-peruen-epoca-de-pandemia/

Hudák, M., Kianickova, E., \& Madlenák, R. (2017). The importance of e-mail marketing in e-commerce [La importancia del marketing por correo electrónico en el comercio electrónico]. Procedia Engineering, 192, 342-347. https://doi. org/10.1016/j.proeng.2017.06.059

Išoraitè, M., \& Miniotienè, N. (2018). Electronic Commerce: Theory and Practice [Comercio electrónico: teoría y práctica]. IJBE: Integrated Journal of Business and Economics, 2(2), 73-79. http://doi.org/10.5281/zenodo.1258437

Kannan, P., \& Li, H. (2017). Digital Marketing: A Framework, Review and Research Agenda [Marketing digital: un marco, una revisión y una agenda de investigación]. International Journal of Research in Marketing, 43(1), 22-45. https:// doi.org/10.1016/j.ijresmar.2016.11.006

Kumar, R. (2011). Research Methodology a Step- by guide for beginners 3 (3rd ed.). Mixed Sources.

Kütz, M. (2016). Introduction to E-Commerce: Combining Business and Information Technology [Introducción al comercio electrónico: combinación de tecnología empresarial y de la información]. Bookboon. https://irp-cdn.multiscreensite.com/1c74f035/files/uploaded/introduction-to-e-commerce.pdf

Matas, J., Medina, M., Fernández, D., \& Aguilar, D. (2016). Análisis del comercio electrónico y su aporte en las transacciones internacionales en la región Junín, Perú. Apuntes de Ciencia \& Sociedad, 6(2), 100-107. https://doi.org/10.18259/ acs. 2016017
Mucha, M. (2018). El comercio electrónico y su relación con el nivel de ventas de las Pymes productoras de calzado, de la Provincia de Trujillo. SCIÉNDO, 21(2), 217-223. https://doi. org/10.17268/sciendo.2018.023

Oliveira, T., Alhinho, M., Rita, P., \& Dhillon, G. (2017). Modelling and testing consumer trust dimensions in e-commerce [Modelar y probar las dimensiones de la confianza del consumidor en el comercio electrónico. Computers in $\mathrm{Hu}$ man Behavior, 71(1), 153-164. https://doi.org/10.1016/j.chb.2017.01.050

Piñeiro, T., \& Martínez, X. (2016). Understanding digital marketing-basics and actions. En C. Machado, \& P. Davim, MBA: Theory and application of business and management principles (págs. 3767). Springer. http://dx.doi.org/10.1007/9783-319-28281-7_2

Reiro, T. (2016). Nonexperimental research: strengths, weaknesses and issues of precision. European Journal of Training and Development, 40(8/9), 676-690. https://doi.org/10.1108/ EJTD-07-2015-0058

Saura, J. (2020). Using Data Sciences in Digital Marketing: Framework, methods, and performance metrics [Uso de las ciencias de datos en el marketing digital: marco, métodos y métricas de rendimiento]. Journal of Innovation \& Knowledge, 6(2), 92-102. https://doi.org/10.1016/j. jik.2020.08.001

Rungsrisawat, S., Sriyakul, T., \& Jermsittiparsert, K. (2019). The Era of e-Commerce \& Online Marketing: Risks Associated with Online Shopping [La era del comercio electrónico y el marketing online: riesgos asociados con las compras online]. International Journal of Innovation, Creativity and Change, 8(8), 201-221. https://www. ijicc.net/images/vol8iss8/8813_R1ungsrisawat_2019_E_R.pdf

Villa, E., Ruiz, L., Valencia, A., \& Picón, E. (2018). Electronic Commerce: Factors Involved in its Adoption from a Bibliometric Analysis [Comercio electrónico: factores involucrados en su adopción a partir de un análisis bibliométrico]. Journal of theoretical and applied electronic commerce research, 13(1) 39-70. http://dx.doi. org/10.4067/S0718-18762018000100104 